July 12,2018

\title{
D-BRANE INTERACTION IN THE TYPE IIB MATRIX MODEL
}

\author{
Bhabani Prasad Mandal and Subir Mukhopadhyay \\ Institute of Physics, Sachivalaya Marg, \\ Bhubaneswar-751005, India, \\ email: bpm,subir@iop.ren.nic.in
}

\begin{abstract}
We calculate the potential between bound states of D-branes of different dimension in IIB matrix model upto one loop order and find nice agreement with the open string calculations in short and large distance limit. We also consider the scattering of bound states of D-branes, calculate the scattering phase shift and analyze the effective potential in different limits.
\end{abstract}




\section{INTRODUCTION}

Recently, a non-perturbative formulation (matrix theory) [四] has been proposed for the M-theory at infinite momentum frame. Making use of the fact, that at infinite momentum frame all other degrees of freedom with finite momentum get decoupled, the M-theory has been shown to be described by that of a system of large number of D-particles. The theory is given by a large- $N$ super Yang-Mills $(\mathrm{SYM})$ theory in $(0+1)$ dimension. A number of issues have been tested such as supergraviton spectrum, D-brane interaction and various kinds of compactifications [1] 7].

This model has inspired another large- $N$ super Yang-Mills theory in $(0+0)$ dimension [8] which is proposed to describe the non perturbative physics of type IIB string. It has its origin in the fact that a large- $N$ reduced SYM theory describes string at a proper double scaling limit [9]. However, a closer look reveals that this is the theory of D-instanton (apart from a chemical potential term in the action) and one can use a similar infinite momentum frame argument to arrive at this theory [10]. This theory, by compactification on one circle and a subsequent wick rotation leads to the BFSS matrix model [1]. The massless spectrum and the light-cone string theory can be recovered from this model [8, 99,11].

In this paper, we shall study the interaction of the D-branes in the latter formulation which, among other things, serves as a consistency check for the type IIB matrix theory and helps to understand the relation between these two matrix theories.

The D-branes are the BPS states of the string theory which couple to the RR fields. Usually a p D-brane couple to a $(\mathrm{p}+1)$ form potential. By virtue of the interaction term $\int C \wedge \operatorname{tr}\left(e^{(i F)}\right)$, a p-brane with electro-magnetic field turned on in its world volume can interact with a brane of lower dimension and thus can form a non-threshold bound states [7]. These composite objects with large electric or magnetic field turned on in its world volume, i.e. a D-brane coupled to a large number of lower dimensional D-branes, come out naturally in the matrix theory as the classical solutions of the equation of motion. So from Matrix theory one can study the interactions between these D-branes. Since the Matrix 
theory describes the interaction mediated through the open string, it should reproduce the short distance behavior. Further, due to the presence of a large number of lower dimensional D-branes it should also reproduce the long distance limit.

In the BFSS theory [1], the interaction among D-branes has been studied in one loop order of the matrix theory leading to a satisfactory agreement with the open string calculation upto one loop both at short and long distance limit [5]. In the IKKT theory [8], the long distance limit of the interaction potential for some configurations breaking half of the SUSY, has been compared with the result obtained from the Born-Infeld action and nice agreement

has been reached [13]. The short distance limit has also been analyzed in [14,15 but the lower dimensional branes have not been considered.

Here we shall study the potential and the scattering phase shift from the IIB matrix theory and compare the results with those obtained from the open and the closed string calculations.

The plan of this paper is as follows. In the next section we will discuss some preliminaries concerning the IKKT model. Then in the two following sections the potential energy and the scattering between two D-branes of odd dimensions will be discussed. Finally we will conclude discussing our results and possible further extensions.

\section{PRELIMINARY}

In this section, we describe the necessary preliminaries required for the study of interaction among D-branes in the type IIB matrix model [8]. Let us start with the action which is obtained by reduction of ten dimensional Euclidean SYM theory with $S U(N)$ gauge group reduced to zero dimension.

$$
S=\alpha\left\{-\frac{1}{4} \operatorname{Tr}\left[A_{\mu}, A_{\nu}\right]^{2}-\frac{1}{2} \operatorname{Tr}\left(\bar{\psi} \Gamma^{\mu}\left[A_{\mu}, \psi\right]\right)\right\}+\beta N
$$

where $A_{\mu}$ and $\psi$ are gauge fields in the $S U(N)$ adjoint and so we can consider them to be $N \times N$ Hermitian matrices. The parameter $N$ is considered to be a dynamical variable, $\alpha$ 
and $\beta$ are related to the string coupling. The third term implies that the sum over $N$ in partition function should be taken with the weight factor $e^{-\beta N}$. Later the sum over $N$ is replaced by a double scaling limit [9].

This action can be viewed either as obtained from type IIB theory by taking the Schield gauge [8] or as the action for $N$ D-instantons [10] apart from the last term. It has the manifest Lorentz invariance and $N=2$ supersymmetry. The SUSY transformations are given by

$$
\begin{aligned}
\delta^{(1)} \psi & =\frac{i}{2}\left[A_{\mu}, A_{\nu}\right] \Gamma^{\mu \nu} \epsilon, \quad \delta^{(2)} \psi=\xi \\
\delta^{(1)} A_{\mu} & =\bar{\epsilon} \Gamma_{\mu} \psi, \quad \delta^{(2)} A_{\mu}=0 .
\end{aligned}
$$

The equation of motion is given by

$$
\left[A^{\mu},\left[A_{\mu}, A_{\nu}\right]\right]=0 \quad\left[A_{\mu},\left(\Gamma^{\mu} \psi\right)_{\alpha}\right]=0
$$

The solution of the equation of motion correspond to non-perturbative states in IIB theory. In particular, those cases for which $\left[A_{\mu}, A_{\nu}\right]=\mathrm{c}-$ number, are interesting as they preserve half of the supersymmetry and are interpreted as BPS states. The odd p-branes, being BPS states in type IIB theory, are associated with this type of solutions.

So a plausible choice for the p-D-brane solution is

$$
\begin{aligned}
& A_{\mu}^{c l}=B_{\mu}, \quad \text { for } \quad 0 \leq \mu \leq p, \quad \psi=0 \\
& A_{\mu}^{c l}=0 \quad \text { otherwise }
\end{aligned}
$$

where $B_{\mu}$ 's are the $N \times N$ Hermitian matrices satisfying $\left[B_{a}, B_{b}\right]=-i c_{a b} I$, and $a, b=0, \cdots p$ with $c_{a b}$ is a c number.

A consistent supersymmetry algebra is yet to be constructed (an attempt was made in 15]) but the central charge of a p-brane can be taken as [4, 14, 15

$$
Z_{i_{1}, \cdots i_{p}}=\left[A_{0}, A_{i_{1}}\right]\left[A_{i_{2}}, A_{i_{3}}\right] \cdots\left[A_{i_{p-1}}, A_{i_{p}}\right]
$$

which is consistent with this solution. But this means that the p-branes contain non zero electric or magnetic flux and so it actually correspond to a large number of lower dimensional branes coupled to it. 
In general, this solutions represent D-branes of infinite extension. However, we would like to deal with finite ones for the time being. So we wrap the p-brane on a torus of dimensions $L_{i}$. For the sake of convenience of the calculation, let us make use of the Lorentz transformation to cast the $c_{a b}$ in a block diagonal form with eigen values $\omega_{i}$ 's . Then the eigenvalues, related to $L_{i}$ by the relation

$$
2 \pi \omega_{i}=\frac{L_{2 i-2} L_{2 i-1}}{N_{i}}
$$

represents the amount of flux linked with the respective planes.

At the end of the calculation we will take the infinite limit for the $L_{i}$ 's and $N_{i}$ keeping $\omega_{i}$ finite. So we can consider the $B_{\mu}$ 's to be Hermitian operator on some Hilbert space and the matrix index can be taken to be continuous. Further, we, use the Schrödinger representation for the Hilbert space operators $B_{i}$ and write

$$
B_{2 i-2}=i \omega_{i} \partial_{i} ; \quad B_{2 i-1}=q_{i} \quad i, j=1,2 \cdots
$$

where $\partial_{i} \equiv \partial / \partial q_{i}$ and $q_{i}$ represent conjugate operators on the Hilbert space. Finite values of $L_{i}$ 's imply that the eigenvalues of $q_{i}$ are distributed in $\left[-\frac{L_{q}}{2}, \frac{L_{q}}{2}\right]$.

We will consider the interaction between p-branes upto one loop. Summing over the fluctuations and considering only the quadratic terms, the one loop effective action of the matrix model can be written in a compact form [8]

$$
W=\frac{1}{2} \operatorname{Tr} \ln \left(P^{2} \delta_{\mu \nu}-2 i F_{\mu \nu}-\frac{1}{4} \operatorname{Tr} \ln \left(P^{2}+\frac{i}{2} F_{\mu \nu} \Gamma^{\mu \nu}\right)\left(\frac{1+\Gamma_{11}}{2}\right)-T r \ln \left(P^{2}\right)\right.
$$

where $P_{\mu}$ and $F_{\mu \nu}$ are operators acting on the space of Hermitian matrices and are defined by

$$
P_{\mu} X=\left[A_{\mu}^{c l}, X\right], \quad F_{\mu \nu} X=i\left[\left[A_{\mu}^{c l}, A_{\nu}^{c l}\right], X\right]
$$

In other words $P_{\mu}$ and $F_{\mu \nu}$ are the adjoint representation of $A_{\mu}$ and $F_{\mu \nu}$ respectively.

Here we will restrict ourselves to the one loop order. We will get the classical solution corresponding to the configuration and obtain the effective action from (2.8). This analysis can be extended to higher loop orders but that will involve [16] explicit Feynman diagrams and the effective action will be much more complicated. 


\section{POTENTIAL BETWEEN BRANES}

In this section we will consider the potential between two branes of dimensions $p$ and $q$ respectively. A configuration of two parallel branes of same dimension is known to saturate the BPS limit and so the potential is zero. For $p \neq q$ in the generic case it breaks the supersymmetry and so the potential is nonzero. All these have been investigated by usual D-brane analysis [12, 17, 18]. However, in matrix model, instead of getting pure D-branes , the classical solutions represent D-branes with electric and magnetic fluxes turned on in its world volume, which, as stated earlier, represent configurations of D-branes of different dimensions [3,6,7] . Our aim is to carry out similar kind of investigation in the IIB matrix model framework.

We shall consider the two brane configuration as the classical solution of the equations

of motion (2.3). Then we will sum over all fluctuations around it upto one loop order using (2.9) and get the interaction potential as described in Ref. [8].

Let us start with the two branes of $p$ and $q$ dimensions parallel to the $X^{1 \cdots p}$ and $X^{1 \cdots q}$ planes and place them at a distance $\pm b / 2$ along $X^{q+1}$ respectively.

The classical configuration is given by,

$$
\begin{aligned}
A_{i} & =\left(\begin{array}{cc}
B_{i} & 0 \\
0 & B_{i}
\end{array}\right) ; \text { for } 0 \leq i \leq p ; \quad A_{i}=\left(\begin{array}{cc}
B_{i} & 0 \\
0 & 0
\end{array}\right) ; \text { for } p+1 \leq i \leq q ; \\
A_{q+1} & =\frac{b}{2}\left(\begin{array}{cc}
1 & 0 \\
0 & -1
\end{array}\right), A_{i}=0 \text { for } i>q+1,
\end{aligned}
$$

where $B_{i}$ 's are the Hermitian operators on Hilbert spaces mentioned earlier.

So the B's in the Scrodinger representation are given by $B_{2 i-2}=i \omega \partial_{i}, B_{2 i-1}=q_{i}$ for $1 \leq i \leq l$ with the value of $\omega$ is given by $(2.6$.

In order to get the potential we have to sum over the fluctuations using (2.8) around this classical solution (3.1). The first thing we need is the adjoint representation of the operators

corresponding to $A_{\mu}$ given by the action on an arbitrary Hermitian matrix according to the (2.9). This is given by 


$$
\begin{aligned}
P_{2 i-2}\left(\begin{array}{cc}
X & Y \\
Y^{\dagger} & Z
\end{array}\right) & =i \omega_{1}\left(\partial_{i}^{1}+\partial_{i}^{2}\right)\left(\begin{array}{cc}
X & Y \\
Y^{\dagger} & Z
\end{array}\right) ; P_{2 i-1}\left(\begin{array}{cc}
X & Y \\
Y^{\dagger} & Z
\end{array}\right)=\left(q_{i}^{1}-q_{i}^{2}\right)\left(\begin{array}{cc}
X & Y \\
Y^{\dagger} & Z
\end{array}\right) \\
P_{q+1}\left(\begin{array}{cc}
X & Y \\
Y^{\dagger} & Z
\end{array}\right) & =b / 2\left(\begin{array}{cc}
0 & Y \\
Y^{\dagger} & Z
\end{array}\right)
\end{aligned}
$$

where each entry is an infinite dimensional matrix e.g. $X=X\left(q^{1}, q^{2}\right)$ and also $P_{1}=$ $i \omega_{1} \partial_{1} \delta\left(q_{1}^{1}-q^{2}-1\right), q_{1}=q^{1} \delta\left(q_{1}^{1}-q_{1}^{2}\right)$ etc. are infinite dimensional matrices.

The adjoint representation of the $F_{\mu \nu}$ can be obtained by using (2.9) from which we see that the only $F_{i, i+1}$ components have non-trivial actions. Further they act only on the matrices of the form $\left(\begin{array}{cc}0 & Y \\ Y^{\dagger} & 0\end{array}\right)$ which correspond to a non-zero eigenvalue. The eigenvalues are $\omega_{i}$ with $k<i \leq l$.

Similarly the eigenvalues of $P^{2}$ can be obtained as

$$
E=2 \sum_{i=1}^{k}\left(p_{i}^{2}+\tilde{q}_{i}^{2}\right)+\sum_{k+1}^{l}\left(p_{i}^{2}+q_{i}^{2}\right)
$$

where $\left[p_{i}, q_{j}\right]=-i \omega_{i} \delta_{i j}$ and other commutators are vanishing. It looks like Hamiltonian of $k$ free particles and $l-k$ oscillators.

Collecting all these three we get the interaction potential from the (2.8). After Simplification it becomes

$$
W=\frac{1}{2} \sum_{1}^{\Delta} \operatorname{Tr} \ln \left(E-2 a_{i}\right)+\operatorname{Tr} \ln \left(E+2 a_{i}\right)-2^{2-\Delta} \sum_{\left\{\lambda_{i}\right\}} \operatorname{Tr} \ln \left(a_{i} \lambda_{i}\right)+(4-\Delta) \ln E
$$

where $\lambda_{i}= \pm 1, \Delta=l-k$ and $a_{i}=\omega_{k+i}$.

In order to compare this with the existing result it is better to write the potential in the form of a proper time integral. Using the identity [14,

$$
\ln a=-\int \frac{d s}{s} \exp (-s a)
$$

we can write the potential as

$$
W=\int \frac{d s}{s} \operatorname{Tr} e^{-s E}\left\{\sum_{1}^{\Delta} \cosh \left(2 s \omega_{i}\right)-4 \prod_{i=1}^{\Delta} \cosh \left(s \omega_{i}\right)+(4-\Delta)\right\}
$$


where $E$ is given by 3.3 .

The trace can be evaluated in a straightforward manner and is given by

$$
\operatorname{Tr} e^{-s E}=\frac{N^{2 k}}{L_{0} \cdots L_{p}}\left(\frac{\pi}{2 s}\right)^{k} \exp -b^{2} s \prod_{i=k+1}^{l}\left(2 \sinh \omega_{i} s\right)^{-1}
$$

Using this we can get

$$
W=C_{p} \prod_{i=1}^{k} \frac{1}{\left|2 \pi \omega_{i}\right|^{2}} \int \frac{d s}{s} \exp \left(-b^{2} s\right)\left(\frac{\pi}{2 s} \cdot \frac{\sum_{i=k+1}^{l} \cosh \left(2 s \omega_{i}\right)-4 \prod_{k+1}^{l} \cosh \left(s \omega_{i}\right)+4-\Delta}{\prod_{i=k+1}^{l} 2 \sinh \left(\omega_{i} s\right)}\right.
$$

where $C_{p}$ is the volume of the hyperplane which is common to both the branes.

Note that this expression is almost identical to the expression of the phase shift obtained in the scattering of D-particle and 4-branes in [7]. Only difference is that they consider the scattering while here we are considering the static potential. This confirms the T-duality between the two matrix models.

As mentioned earlier this represents the potential between $p$ and $q$-branes with stack of lower dimensional branes attached to these. In order to compare this with the result obtained from open string calculation upto one loop we consider the specific brane configurations.

For the sake of brevity let us consider the 3-brane and 1-brane configuration. So we put $q=3$ and $p=1$. The 3 -brane has a magnetic field turned on in its world volume on the $X^{23}$ plane with strength $\int_{C_{23}} F \equiv \omega_{2}$. Besides, both the 3 and the 1-brane has an electric field in their world volume with $\int_{C_{01}} F \equiv \omega_{1}$. So the 3 -brane, considered in this matrix model actually represents a bound state of a 3-brane, $\frac{L_{2} L_{3}}{2 \pi \omega_{2}}$ D-strings along the $X^{1}$ and $\frac{L_{0} L_{1}}{2 \pi \omega_{1}}$ D-instantons and similarly the 1-brane represents a bound state of a 1-brane with $\frac{L_{0} L_{1}}{2 \pi \omega_{1}}$ D-instantons.

In this case the potential is reduced to

$$
W=L_{1} \frac{1}{\left|2 \pi \omega_{1}\right|^{2}} \int \frac{d s}{s} \exp \left(-b^{2} s\right)\left(\frac{\pi}{2 s}\right) \cdot \frac{\cosh \left(2 s \omega_{2}\right)-4 \cosh \left(s \omega_{2}\right)+3}{2 \sinh \left(\omega_{2} s\right)}
$$

Note that the contribution from the common field always comes through the free particle term in the $P^{2}$ eigenvalue $(3.3)$ and so always gives rise to an overall factor . The tachyonic instability [19], like that in the BFSS model [7] is also present for very small separation. 
We consider the potential for the above configuration already calculated from open string theory upto one loop. A 3-brane with electric field $F_{1}$ along $X^{1}$ and a magnetic field $F_{2}$ along $X^{23}$ plane interacts with a 1-brane with the same electric field in its world volume through the term $\int C \wedge \operatorname{tr}\left(e^{(i F)}\right)$.

The potential is given by [12]

$$
A=\frac{L\left(1+F_{1}^{2}\right)}{2 \pi} \int \frac{d s}{s} \frac{e^{-b^{2} s}}{4 \pi s} B \times J
$$

where $B$ and $J$ are the contribution from the bosonic and the fermionic term respectively and the prefactor takes care of the common electric field. Also we have taken $\alpha^{\prime}=\frac{1}{2 \pi}$.

$B$ and $J$ are given by

$$
\begin{aligned}
B & =\frac{1}{2} f_{1}^{-4} \Theta_{1}^{-1}(i \epsilon s) \\
J & =\left(-f_{2}^{4} \Theta_{2}(i \epsilon s)+f_{3}^{4} \Theta_{3}(i \epsilon s)+f_{4}^{4} \Theta_{4}(i \epsilon s)\right)
\end{aligned}
$$

where $\epsilon$ is related to the magnetic field by $\tan (\pi \epsilon)=F_{2}$

The matrix model describes the interaction through the open string and so let us consider the short distance limit of this potential.

If we write $\epsilon_{i}=\frac{\pi}{2}-\pi \omega_{i}$ where $\tan \left(\pi \epsilon_{i}\right)=F_{i}$ and consider the contribution of the massless modes only the in the limit of large fields i.e. small $\omega$ the potential can be written as

$$
W=L_{1} \frac{1}{\left|2 \pi \omega_{1}\right|^{2}} \int \frac{d s}{s} \exp \left(-b^{2} s\right)\left(\frac{\pi}{2 s}\right) \cdot \frac{\cosh \left(2 s \omega_{2}\right)-4 \cosh \left(s \omega_{2}\right)+3}{2 \sinh \left(\omega_{2} s\right)}
$$

which is precisely the result obtained from the matrix model.

The comparison also makes it clear that, at least at the short distance limit, the bosonic contribution comes from the $t r e^{-s E}$ term while the $(-1)^{F}$ of the R sector contribution comes from the second term in the braces of (3.6). It is interesting that how the latter will flip sign in the case of the brane-antibrane potential.

We can now calculate the long range potential from the matrix model calculation for $p$, $q$-branes and compare with that obtained in string theoretic calculation. A straightforward approximation for the large value of $b$ in the expression for potential in (3.8) leads to the expression 


$$
\frac{\pi k}{2} \frac{\left[\frac{1}{2} \sum_{i=k+1}^{l} \omega_{i}^{4}-\sum_{i<j} \omega_{1}^{2} \omega_{j}^{2}\right]}{2^{l-k} \prod_{i=k+1}^{l} \omega_{i}} \Gamma(4-l) b^{2 l-8} \equiv V(b)
$$

Now we will see how this potential depends on $\mathrm{b}$ for the specific cases .

For 1-brane and 3-brane interaction, $V(b)=\frac{\pi^{4}}{8} \frac{1}{F_{23}^{3} b^{4}}$ which is a repulsive potential. Similarly for the case of 3-brane and 5-brane interaction, $V(b)=\frac{\pi^{4}}{8} \frac{1}{F_{45}^{3} b^{4}}$. Something interesting happens for the interaction between 1-brane and 5-brane. In that case $V(b)=\frac{\pi\left(\omega_{2}^{2}-\omega_{3}^{2}\right)^{2}}{16 \omega_{2} \omega_{3}}$ $V(b)=\frac{\pi^{3}}{b^{4}} \frac{\left(F_{23}^{2}-F_{45}^{2}\right)}{F_{23}^{3} F_{45}^{3}}$. and the potential vanishes for $F_{23}=F_{45}$. This is because when the magnetic fields in the $X^{23}$ and $X^{34}$ planes are equal the configuration becomes a BPS state and so there is no force between them.

Other D-brane bound states can be analysed in a similar manner and those also lead to a similar kind of agreement. Also one can consider the D-branes oriented orthogonally to each other. Further all these results also match with those obtained from BFSS model which is an evidence for the duality between the matrix theories.

\section{SCATTERING OF BRANES}

In this section, we will consider the interaction between two moving branes of different dimensions. Again such a configuration is not a BPS state and breaks the supersymmetry. We will calculate the phase shift of one brane (of lower dimension) while passing by the other in an eikonal approximation [6, [] in the matrix model and then compare with the results obtained by D-brane technique [17, 18].

Let us consider two branes of dimension $p$ and $q$ parallel to the $X^{1 \cdots p}$ and $X^{1 \cdots q}$ hyperplanes moving parallel to $X^{q+1}$ with a relative velocity $v$ at a relative separation $b$ along $X^{q+2}$ axis. The classical solutions corresponding to this configuration can easily be obtained by boosting the static solutions.

These solutions are:

$$
A_{0}=\left(\begin{array}{cc}
B_{0} \cosh \epsilon & 0 \\
0 & B_{0} \cosh \epsilon
\end{array}\right) ; \quad A_{i}=\left(\begin{array}{cc}
B_{i} & 0 \\
0 & B_{i}
\end{array}\right) ; \text { for } 1 \leq i \leq p
$$




$$
\begin{aligned}
A_{i} & =\left(\begin{array}{cc}
B_{i} & 0 \\
0 & 0
\end{array}\right), \quad \text { for } p<i \leq q \quad A_{q+1}=\left(\begin{array}{cc}
B_{0} \sinh \epsilon & 0 \\
0 & B_{0} \sinh \epsilon
\end{array}\right) ; \\
A_{q+2} & =\frac{b}{2}\left(\begin{array}{cc}
1 & 0 \\
0 & -1
\end{array}\right) ; \quad A_{i}=0 \text { for } i \geq q+3 .
\end{aligned}
$$

where $B_{i}$ 's have the usual meaning mentioned earlier, $v \equiv \tanh \epsilon$ and $\epsilon$ is the boosting angle.

Now we consider the action of the solution on Hermitian matrices in the adjoint representation. This time the representations are more complicated than the previous one due to the presence of boost. The explicit forms are

$$
\begin{aligned}
P_{0}\left(\begin{array}{cc}
X & Y \\
Y^{\dagger} & Z
\end{array}\right) & =i \omega_{1} \cosh \epsilon\left(\partial_{1}^{1}+\partial_{1}^{2}\right)\left(\begin{array}{cc}
X & Y \\
Y^{\dagger} & Z
\end{array}\right) ; \quad P_{1}\left(\begin{array}{cc}
X & Y \\
Y^{\dagger} & Z
\end{array}\right)=\left(q_{1}^{1}-q_{1}^{2}\right)\left(\begin{array}{cc}
X & Y \\
Y^{\dagger} & Z
\end{array}\right) ; \\
P_{q+1}\left(\begin{array}{cc}
X & Y \\
Y^{\dagger} & Z
\end{array}\right) & =i \omega_{1} \sinh \epsilon\left(\begin{array}{cc}
\left(\partial_{1}^{1}+\partial_{1}^{2}\right) X & \left(\partial_{1}^{1}-\partial_{1}^{2}\right) Y \\
-\left(\partial_{1}^{1}-\partial_{1}^{2}\right) Y^{\dagger} & -\left(\partial_{1}^{1}+\partial_{1}^{2}\right) Z
\end{array}\right) ; \\
P_{p+1}\left(\begin{array}{cc}
X & Y \\
Y^{\dagger} & Z
\end{array}\right) & =i \omega_{k_{1}+1}\left(\begin{array}{cc}
\left.\left(\partial_{k+1}^{1}\right)+\partial_{k+1}^{2}\right) X & \partial_{k+1}^{1} Y \\
\partial_{k+1}^{2} Y^{\dagger} & 0
\end{array}\right) ; \\
P_{p+2}\left(\begin{array}{cc}
X & Y \\
Y^{\dagger} & Z
\end{array}\right) & =\left(\begin{array}{cc}
\left(q_{k+1}^{1}-q_{k+1}^{2}\right) X & q_{k+1}^{1} Y \\
-q_{k+1}^{2} Y^{\dagger} & 0
\end{array}\right) ; P_{q+2}\left(\begin{array}{cc}
X & Y \\
Y^{\dagger} & Z
\end{array}\right)=b\left(\begin{array}{cc}
0 & Y \\
Y^{\dagger} & Z
\end{array}\right) ; \\
P_{i}\left(\begin{array}{cc}
X & Y \\
Y^{\dagger} & Z
\end{array}\right) & =0 ; i \geq q+3
\end{aligned}
$$

where $q^{1}$ and $q^{2}$ are the first and the second arguments of the infinite dimensional matrices.

We evaluate the action of $F_{i j}$ in the adjoint representation and it has the non-zero action only on the matrices $Y$.

$F_{1, q+1}\left(\begin{array}{cc}X & Y \\ Y^{\dagger} & Z\end{array}\right)=2 \omega_{1} \sinh \epsilon\left(\begin{array}{cc}0 & Y \\ Y^{\dagger} & 0\end{array}\right) ; F_{2 i-2,2 i}\left(\begin{array}{cc}X & Y \\ Y^{\dagger} & Z\end{array}\right)=-i \omega_{i}\left(\begin{array}{cc}0 & Y \\ -Y^{\dagger} & 0\end{array}\right) ; i=k+1 \cdots q$

Therefore $F_{i j}$ gives nontrivial results only when it acts on $Y$. So we plug in the above expressions in the equation (3.8) and get the phase shift to be 


$$
\begin{aligned}
R e W & =\int_{0}^{\infty} \frac{d s}{s} \operatorname{Tr} e^{-s E}\left(\cosh \left(2 s \omega_{1} \sinh \epsilon\right)+\sum_{k+1}^{l} \cosh \left(2 s \omega_{i}\right)\right. \\
& \left.-2^{1-\Delta_{2}} 2 \cosh \left(2 s \omega_{1} \sinh \epsilon\right) \prod_{k+1}^{l} 2 \cosh \left(s \omega_{i}\right)+3-\Delta\right)
\end{aligned}
$$

where $\Delta=l-k$.

The eigen values of $P^{2}$ in the adjoint representation can be obtained in a straightforward manner which again represents the Hamiltonian of some free particles and some harmonic oscillators.

$$
E=2 p_{1}^{2} \cosh ^{2} \epsilon+2 \sum_{i=2}^{k}\left(p_{i}^{2}+\tilde{q}_{i}^{2}\right)+\sum_{i=k+1}^{l}\left[p_{i}^{2}+q_{i}^{2}\right]+2\left[p_{1}^{2} \sinh ^{2} \epsilon+q_{i}^{2}\right]+b^{2}
$$

where $\left[p_{i}, q_{j}\right]=-i \omega_{i} \delta_{i j}$ for $k<i, j \leq l$ and all other commutators vanish.

Now we calculate $\operatorname{Tr} e^{-s E}$ as

$$
\operatorname{Tr} e^{-s E}=\frac{N^{2 k-1} L_{1}}{L_{0} L_{1} \cdots L_{p}}\left(\frac{\pi}{2 s}\right)^{\frac{p}{2}} \frac{1}{\cosh \epsilon} \frac{e^{-b^{2} s}}{2 \sinh \left(s \omega_{1} \sinh \epsilon\right) \prod_{i=k+1}^{l} 2 \sinh \left(2 s \omega_{i}\right)}
$$

Substituting this expression for Trace in 3.6 we get

$$
R e W=-V_{p} 2 \pi \omega_{i} \prod_{i}^{k} \frac{1}{\left(2 \pi \omega_{i}\right)^{2}} \int_{0}^{\infty} \frac{d s}{s} e^{-b^{2} s}\left(\frac{\pi}{2 s}\right)^{\frac{p}{2}}
$$

So the expression of the phase shift can be written as

$$
\begin{aligned}
& R e W=-V_{p} 2^{\frac{-p}{2}} \omega_{1} \prod_{i=1}^{k} \frac{1}{\omega_{i}^{2}} \int_{0}^{\infty} \frac{d s}{s} e^{-b^{2} S}(4 \pi s)^{\frac{-p}{2}} \\
& \times \frac{\cosh \left(2 s \omega_{1} \sinh \epsilon\right)+\sum_{k+1}^{l} \cosh \left(2 s \omega_{i}\right)-2^{1-\Delta} 2 \cosh \left(s \omega_{1} \sinh \epsilon\right) \prod_{k+1}^{l} 2 \cosh \left(s \omega_{i}\right)+3-\Delta}{\cosh \epsilon 2 \sinh \left(2 s \omega_{1} \sinh \epsilon\right) \prod_{i=k+1}^{l} 2 \sinh \left(s \omega_{i}\right)}
\end{aligned}
$$

where $V_{p}$ represents the space time volume of the $p$-brane.

In order to compare with the open string result it is better to consider a specific configuration. We consider a simple one - in between a 3-brane and a 1-brane. So we take $p=1$ and $q=3$. Also as in the last section the 3 -brane has non-zero magnetic field along the $X^{23}$ plane and each of the branes has an electric field in $X^{1}$ direction.

Then the phase shift gets reduced to 


$$
\begin{aligned}
& \operatorname{ReW}=-\frac{V_{1}}{4 \sqrt{2}} \omega_{1} \frac{1}{\omega_{1}^{2}} \int_{0}^{\infty} \frac{d s}{s} e^{-b^{2} s}(4 \pi s)^{\frac{-1}{2}} \\
& \times \frac{2 \cosh \left(2 s \omega_{1} \sinh \epsilon\right)+2 \cosh \left(2 s \omega_{2}\right)-8 \cosh \left(s \omega_{1} \sinh \epsilon\right) \cosh \left(s \omega_{2}\right)+4}{2 \cosh \epsilon \sinh \left(2 s \omega_{1} \sinh \epsilon\right) 2 \sinh \left(s \omega_{2}\right)}
\end{aligned}
$$

It is difficult to obtain the scattering phase shift between two D-brane configurations with electric field in their world volume from the open string calculation. The presence of the electric field and the velocity mixes up the coordinates and it is complicated to get a basis in which the energy momentum tensor can be diagonalized. But we have seen, in the case of the potential and also in the cases of scattering with a magnetic field, that the matrix model result gives the correct short distance behavior. So we can demand that the matrix model gives the phase shift for branes with electric fields in world volume, at least at the limit of infinite field strength.

In the case of scattering of p, q-branes we can also calculate the long range potential from matrix model calculations. This can be obtain from (4.8) by considering large $b$ approximation.

$$
\frac{\tanh \epsilon \prod_{i=k+1}^{l} \frac{1}{a_{i}} \sum_{i=k}^{l} a_{i}^{4}-2 \sum_{i<j} a_{i}^{2} a_{j}^{2}}{a_{k} \prod a_{i} b^{7-q}} \equiv V^{s}(b)
$$

where $a_{i}=\omega_{i}$ for $k+1 \leq i<l$ and $a_{k}=\omega_{1} \sinh \epsilon$. This is the effective potential responsible for the scattering. In the case of two branes of same dimension it gets reduced to

$$
V^{s}(b, \epsilon)=\omega_{1}^{2} \sinh ^{2} \epsilon \tanh ^{2} \epsilon b^{q-7}
$$

In the low velocity limit the leading order term is $V(b, v)=\frac{\pi^{2} v^{4}}{F_{01}^{2} b^{7-p}}$. So it reproduces the correct leading order behavior of the potential. For electric field becomes infinite, the boundary conditions becomes Dirichlet and so the potential vanishes.

Now let us consider the specific cases: For 1-brane and 3-brane scattering case ,

$$
V^{s}(b, \epsilon)=\frac{\tanh \epsilon\left(\omega_{1}^{2} \sinh ^{2} \epsilon-\omega_{2}^{2}\right)^{2}}{\omega_{1} \omega_{2}^{2} \sinh \epsilon} b^{-4} .
$$

Therefore for $\sinh \epsilon=\frac{F_{01}}{F_{34}}$ again the potential will vanish giving rise to BPS state. In the low velocity limit, $V(b, v) \sim \frac{\pi F_{01}}{F_{34}^{2} b^{4}}\left(1-\frac{F_{34}^{2}}{F_{01}^{2}} v\right)^{2}$. Similar interpretation will hold good for the case of 3-brane scattering with 5-brane. 
For $p=1, q=5$ the potential that corresponds to the phase shift is given by

$$
\begin{aligned}
V(\epsilon, b) \sim \frac{\tanh \epsilon}{\omega_{1}^{2} \sinh \epsilon} \times \frac{1}{\omega_{2}^{3} \omega_{3}^{2}}\left(\omega_{1} \sinh \epsilon-\omega_{2}+\omega_{3}\right) & \left(\omega_{1} \sinh \epsilon+\omega_{2}-\omega_{3}\right) \\
\times & \left(\omega_{1} \sinh \epsilon-\omega_{2}-\omega_{3}\right)\left(\omega_{1} \sinh \epsilon+\omega_{2}+\omega_{3}\right) \times b^{-2}
\end{aligned}
$$

So for $\sinh \epsilon= \pm \frac{\omega_{2}-\omega_{3}}{\omega_{1}}, \pm \frac{\omega_{2}+\omega_{3}}{\omega_{1}}$ the potential will vanish.

In the low velocity limit it becomes

$$
V(b, v)=\frac{\omega_{1}}{\left(\omega_{1} \omega_{2} \omega^{3}\right)^{2}}\left[\omega_{1}^{2} v^{2}-\left(\omega_{2}-\omega_{3}\right)^{2}\right]\left[\omega_{1}^{2} v^{2}-\left(\omega_{2}+\omega_{3}\right)^{2}\right]
$$

It is interesting that here the potential changes its sign twice with the increase of the velocity.

The matrix model results, as mentioned earlier, is expected to reproduce the short distance behavior. For these cases of bound states of D-branes this should be true at least in the limit of the large electromagnetic field which we have checked in the case of the potential. On the basis of the presence of large field we can similarly argue [6:7] that the long distance limit should also be reproduced reliably. It will be interesting to check these scattering of the bound states of D-branes (with electric field) in the closed string theory.

\section{DISCUSSION}

In this paper, we have discussed the potential between D-branes with electromagnetic fields turned on in its world volume in the matrix model framework. This sort of configuration correspond to the stack of lower dimensional branes attached to it and thus forming a non-threshold bound state. Such a configuration, usually, does not saturate the BPS bound but we have seen that they correspond to the classical solutions of the matrix model which breaks half of the supersymmetry. The fact that the number of the lower dimensional branes is infinity makes this possible.

The interaction of these kind of objects have been discussed in [12] from the open string calculation. We have seen that our result agrees with those obtained from open string 
calculation at the short distance limit. This is expected since the matrix model takes care of the massless modes of the open strings and the open strings are known to dominate the dynamics at the short distance limit. We have matched our result with the long distance limit also which is dominated by the closed strings. This is again due to the fact that the electromagnetic fluxes are very large.

Apart from getting agreement with the string theory, we have also noted the similarity between the potential between the odd and the even dimensional branes obtained from the two different matrix models which is an evidence for the duality between the two matrix models. This is also expected since they describe the IIA and IIB theories and the dynamics are also of the D-particles and the D-instantons which are T-dual to each other.

We have also calculated the scattering phase shift between branes of odd dimensions with electromagnetic fields. Due to the presence of the electric fields i.e the D-instantons attached to the branes, this type of scattering is difficult to study in the open string calculation. On the other hand it is easier to study it in the matrix model framework. On the basis of other agreements we can propose the result to be consistent with the true short distance behavior at the limit of large field. The long distance limit is also interesting and for a few choices of velocities the potential obtained from the phase shift vanishes, signaling existence of BPS saturation. A similar investigation in the closed string theory may be interesting.

Finally, we have considered the trivial classical solution of the matrix model. There are non-trivial solutions, such as instanton solutions which correspond to other D-brane configurations and the the dynamics of these kind of objects can be studied in a similar manner. Also there are anti branes whose interaction with the branes may be studied in this framework. However as mentioned earlier in such a case the fermionic contribution should flip its sign and it is not apparent how it will come about. By using the T-duality argument in the matrix model one can study the interaction between the D-branes and the NS 5-branes of type IIB theory in away similar to that used in [20]. Also it will be interesting if the pure D-brane or a D-brane with finite flux turned on can be constructed and studied in this matrix model framework. 
Acknowlegement: It is a plesure to thank Dr. A. Kumar for useful discussions and for careful reading of the manuscript. We would also like to thank K. Roy for useful discussions and suggestions. 


\section{REFERENCES}

[1] T. Banks, W. Fischler, S.H. Shenker and L. Sussikind, "M theory as matrix model: A conjecture," Phys. Rev. D 55,5112 (1997), hep-th/9610043.

[2] W. Fischler, E. Halyo, A. Rajaram and L. Susskind, " The Incredible Shrining Torus", hep-th/9703102 ; R. Dijkgraff, E. Verlinde and H. Verlinde, "Matrix String thory", hep-th/9704018. T Banks, N. Seiberg and L. Sussikind Nucl. Phys. B 49741 (1997).

[3] O. J. Ganor, S. R. Ramgoolam, W. Taylor IV, Nucl. Phys. B 492, 191 (1997).

[4] T. Banks, N. Seiberg and S.H. Shenker, " Branes from matrices" hep-th/9612157.

[5] B. Aharony and M. Berkooz, " Membrane dynamics in M(atrix) theory," hepth/9611225;

[6] G. Lifschytz and S.D. Mathur, " Supersymmetry and membrance interaction in M(atrix) theory," hep-th/9612087.

[7] G. Lifschytz , " Four-brane and six-brane interactions in M(atrix) theory ," hepth/9612223.

[8] N. Ishibash, H. Kawai, Y. Kitazawa and A. Tsuchiya, " A large- N reduced model as superstring," hep-th/9612115.

[9] B. Sethiapalan, " Fundamental strings and D-strings in the type IIB matrix Model," hep-th/9703133.

[10] A.A. Tseytlin, Phys. Rev. Lett. 78 , 1864 (1997), hep-th/9612164.

[11] M. Li, " Strings from type IIB matrices," hep-th/9612222.

[12] G. Lyfshitz , " Comparing D-branes to black branes," hep-th/9604156; "probing bound states of D-branes" hep-th/9610125.

[13] I. Chepelov and A.A. Tseytlin, " Interaction of type IIB D-branes from D-instanton," 
hep-th/9705120.

[14] A. Fayyazuddin, Y.Makenko, P. Olesen, D.J. Smith and K. Zarembo," Nucl. Phys. B 499, 159-182 (1997), hep-th/9703038.

[15] I. Chepelov, Y. Makeenko and K. Zarembo," Properties of D-branes in matrix model of IIB superstring," hep-th/9701151; A. Fayyazuddin and D. J. Smith, Mod. Phy. Lett. A12 1447-1454, (1997).

[16] K. Becker and M. Becker, " A two loop test of M(atrix) theory," hep-th/9705091.

[17] J. Polchinshi, " TASI lectures on D-branes," hep-th/9611050.

[18] C. Bachas, Phys. Lett. B 374, 37 (1996) ; C. Bachas and M. Porati, Phys. Lett. B 296, 77 (1992); M. B. Green and M. Gutperle, Nucl. Phys. B 476, 484 (1996).

[19] T. Banks and L. Susskind, "Branes-antibrane forces", hep-th/9511194.

[20] G. Lifschytz, " A note on transverse five brane in M(atrix) theory," hep-th/9703201. 